\title{
Sensitivity Modeling of Binary Optical Receivers
}

\author{
Dirk Giggenbach and Ramon Mata-Calvo \\ Institute of Communications and Navigation, German Aerospace Center (DLR), D-82234 Wessling \\ Contact Author: dirk.giggenbach@dlr.de
}

\begin{abstract}
The sensitivity characteristics of optical receiver frontends for high-speed data communications depend on modulation format, detector type, and specific operational constraints. A general mathematical model of the receiver sensitivity that fits to analytical as well as measured data is required to compare different receiver implementations and assess the reliability of data links under varying received power as common in free-space optical communication links. In this paper, a new approach based on Q-factor modelling is presented, compared with analytical receiver models, and applied to a multitude of exemplary receiver implementations. A methodology is introduced to generally apply the model to ideal or practical binary optical receiver frontends.
\end{abstract}

Index Terms - optical receiver frontend sensitivity; Qfactor; sensitivity slope; avalanche photo diode

\section{INTRODUCTION}

While in terrestrial fiber communication systems the optical signal power levels are monitored and well controlled, a variety of environmental parameters do influence the shortand long-term received optical power in long-range FreeSpace Optical (FSO) data links [1]. Such systems can be aeronautical data down-links or satellite up- and down-links [2][3], or even fixed terrestrial point-to-point data connections under varying meteorological conditions [4]. Effects that cause received power variations over several orders of magnitude include atmospheric attenuation (rain, fog, snow), and varying free-space loss caused by changes of the link range in mobile link scenarios. Fast fluctuations are caused by beam pointing errors and scintillations due to atmospheric index-of-refraction turbulence [5]. Under such conditions the receiver performance - in terms of bit-error-rate (BER) versus instantaneous received optical power - is the core parameter for system layout and optimization. Although in high-speed fiber-links, higher modulation formats today are prevailing, in practical FSO only binary modulation formats are of practical use to better cope with the signal level fluctuations mentioned above. Timely examples of such links can be found in the domain of optical space relays and space to ground links. For this scenario, NASA is currently developing technology based on pre-amplified DPSK at 1550nm wavelength [6], whereas ESA is implementing the European Data Relay System (EDRS) based on homodyne BPSK at 1064nm [7] [8].

In our terminology the communication receiver front end (RFE) consists of an input-port receiving the mean optical signal power $\bar{P}_{R x}$ and converting the optically modulated data to electrical signal current. This can be done by simple direct detection through linear photo detectors, or with internal gain (avalanche photo detectors, APD), by self-homodyne-DPSK, or even by a heterodyning receiver employing a local oscillator laser. A subsequent trans-impedance amplifier (TIA) converts and amplifies the electrical signal current to a signal voltage, which is then thresholded into a binary data stream via a limiting amplifier, and can finally be evaluated by means of a bit-error (BER) tester (figure 1).

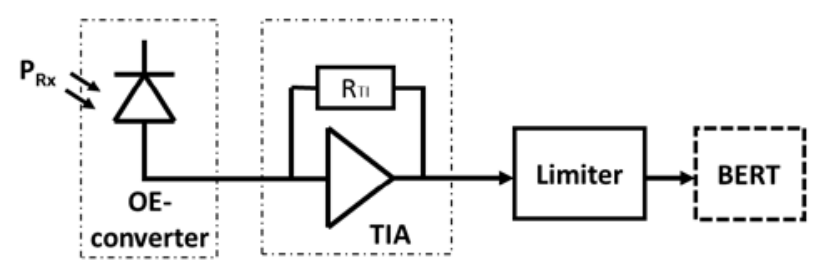

Fig. 1. Generic binary RFE layout.

One can conveniently describe the characteristic of binary RFEs based on the relationship between $\bar{P}_{R x}$, the BERfunction, and its argument $Q$ [9]:

$$
B E R=\frac{1}{2} \cdot \operatorname{erfc}\left(\frac{Q}{\sqrt{2}}\right)=\frac{1}{2} \cdot \operatorname{erfc}\left(\frac{f\left(\bar{P}_{R x}\right)}{\sqrt{2}}\right)
$$

Where $Q$ is be expressed as a pure function of mean received power, $Q=f\left(\bar{P}_{R x}\right)$. Here the functional relation represents all practical implications and non-ideal electronic effects.

We describe in this paper two parameters for qualitative RFE classification and modelling in terms of absolute 
sensitivity and in terms of sensitivity-slope characteristic. The remainder of this paper is structured as follows: In section II the limited applicability of ideal optical RFE models is analyzed. In section III the new receiver modelling scheme is defined, and in section IV its application to measured RFEperformance data is explained and the method is compared with measured performance data. Section $\mathrm{V}$ discusses the results, and table III provides several example applications of the model to measured RFE implementations.

The main objective of this paper is to define a practically applicable methodology to qualitatively assess binary RFE performances without requiring detailed knowledge of implementation-specific technical parameters.

\section{IDEAL RFE-MODELS}

Usually, the theoretical functional relation (1) can be provided for various types of RFEs depending on the dominant noise sources, i.e. thermal noise or shot noise [9][10][11].

In sufficient approximation an optimum receiver bandwidth $B$ equal to half of the channel symbol rate $r$ is assumed $(B=r / 2)$.

In Table I, the three ideal Q-factor equations for modulation with equal fractions of pulse on-time and off-time are summarized: the thermal noise limited Q-factor $Q_{t, 0 o K}$, the shot-noise limited coherent-homodyne $Q_{s, B P S K}[12]$, and the $Q_{s t, O O K}$ when both, thermal and shot noise, are taken into account (as seen in APD-RFEs) [13][14].

TABLE I

CLASSICAL ANALYTICAL RFE MODELS

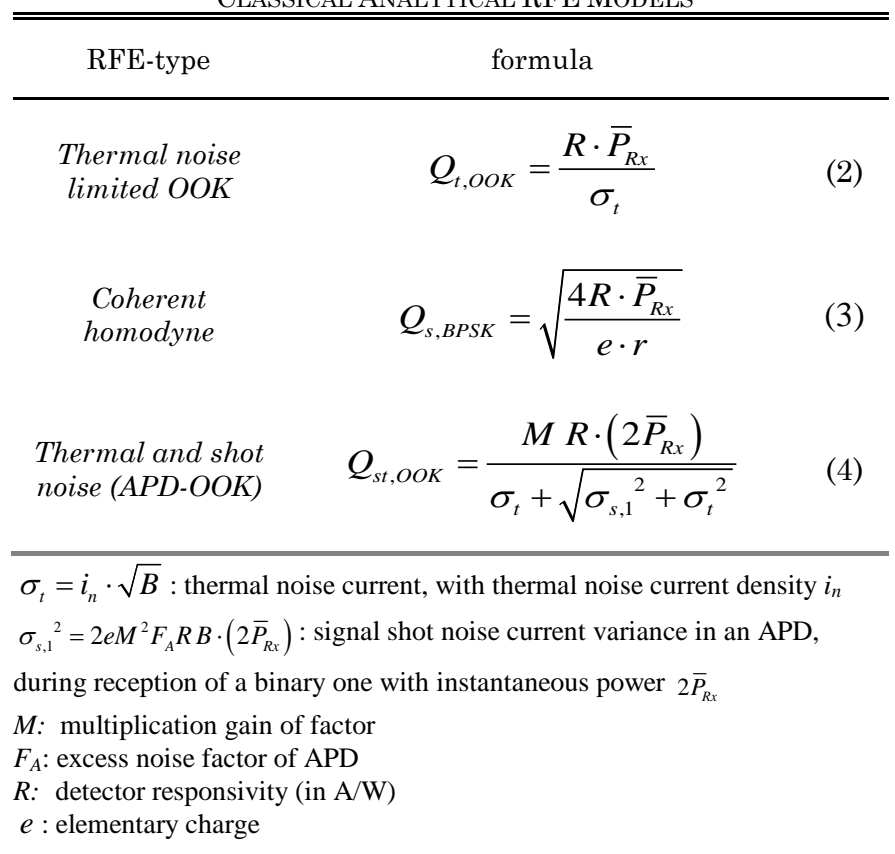

The required mean energy per bit for OOK-NRZ modulation is then:

$$
\bar{E}_{s t, \text { OOK }}(Q)=\frac{Q^{2} M F_{A} e \sqrt{B}+i_{n} Q}{2 M R \sqrt{B}}
$$

For dominating thermal noise, (5) behaves according to the ideal thermal noise limited receiver model with required energy per bit $\bar{E}_{s t, 0 o K} \sim B^{-1 / 2}$. When though shot noise is dominant, $\bar{E}_{s t, \text { оoK }}$ converges to independent behavior from bandwidth. The required energy per bit at shot noise limit with an APD-receiver is still 3dB higher than for the shot noise limited coherent BPSK receiver, since BPSK modulation provides a signal amplitude at both digital ones and zeros [12]. Both shot-noise limited receiver types however exhibit the same rate-independent sensitivity behavior.

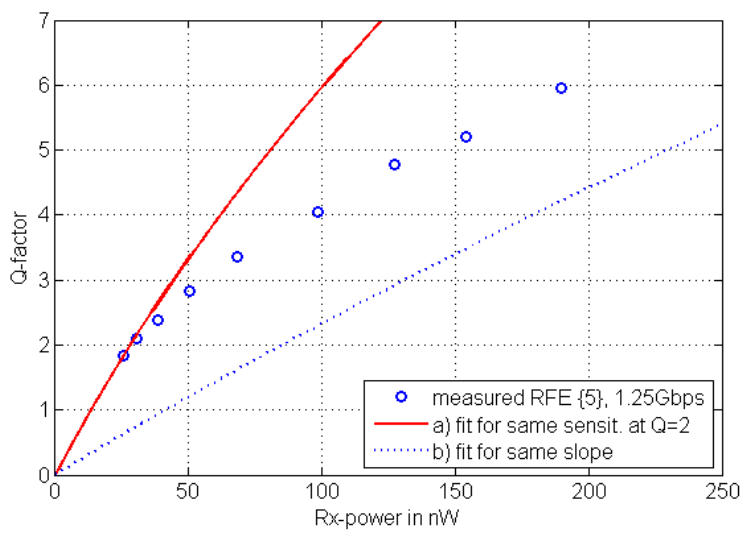

Fig. 2. Measured APD-RFE $\{5\}$ (see Table III) performance compared with sensitivity curves according to (4), showing the deviation of the ideal curves which cannot model the practical RFE characteristics completely, a) to a realistic parameter set that provides the same sensitivity at $Q=2$, and $b$ ) to parameters that provide the same slope as the measured RFE.

Theoretical ideal models of thermal noise limited PIN-RFEs (2) as well as shot-noise limited coherent receivers (3), can model practical RFE sensitivity satisfactorily. Predicting the behavior of APD-receivers (4) (influenced by both thermal and shot noise) however is not accurate, as their characteristics depend on several factors such as semiconductor technology, dark current, modulation extinction ratio, and optimization of the multiplication factor $M$, which again is a function of temperature and received signal power. Fig. 2 demonstrates with an exemplary APD-RFE implementation, that absolute receiver sensitivity (here: required received power for $\mathrm{Q}=2$ ), and its sensitivity slope, hardly can both be modelled sufficiently with a realistic parameter set.

\section{RFE-ModeLLING MethodolOGY}

While the formulas in section II are based on idealized characteristics of thermal and shot noises, they fail in predicting realistic RFE implementations in terms of absolute sensitivity and sensitivity slope. Some RFE-modelling approaches are based on analytical relations between receiver 
parameters to derive the slope of $Q$ around a working point like for an APD-receiver, as in [15]. However such models refer to specific types of RFEs and do not generally account for practical effects. A general useful modelling must be flexible enough to adopt to any measured performance data.

In practice, any RFE-types will exhibit a sensitivity slope between thermal limited and shot-noise limited and thus can be modelled with a scaled power function that allows two degrees of freedom, via its exponent $n$ and the scaling constant $a$ :

$$
Q\left(\bar{P}_{R x}\right)=a \cdot\left(\bar{P}_{R x}\right)^{n_{x, y}}=x \cdot\left(\frac{\bar{P}_{R x}}{\bar{P}_{Q=x}}\right)^{n_{x, y}}
$$

For a reasonable representation, (6) is normalized to a reference power value $\bar{P}_{R x}(Q=x)=\bar{P}_{Q=x}$ from which follows that $a$ will automatically take the value $x /\left(\bar{P}_{Q=x}\right)^{n_{x, y}}$. This normalization allows comparing RFEs of different sensitivity in the same plot of $Q\left(\bar{P}_{R x} / \bar{P}_{Q=x}\right)$, since all curves will cross the coordinate $(1 ; x)$. The exponent $n_{x, y}$ is directly calculated as:

$$
n_{x, y}=\frac{\ln \left(q_{x, y}\right)}{\ln \left(s_{x, y}\right)} \text {, with } s_{x, y}=\frac{\bar{P}_{Q=y}}{\bar{P}_{Q=x}}, q_{x, y}=\frac{y}{x}
$$

$q_{x, y}$ is the ratio between two $Q$ values $x$ and $y$. These values define the reference power thresholds $\bar{P}_{Q=x}$ and $\bar{P}_{Q=y}$ used to calculate the span-parameter, here generally written as $s_{x, y}$.

For analyzing and comparing different RFEs, a practical span is introduced, ranging from the lower bound $P_{Q=2}$ to the upper bound $P_{Q=6}$.

$$
\begin{aligned}
& \bar{P}_{Q=2}=\bar{P}_{R x}(Q=2) \rightarrow \operatorname{BER}\left(\bar{P}_{Q=2}\right)=0.023 \\
& \bar{P}_{Q=6}=\bar{P}_{R x}(Q=6) \rightarrow \operatorname{BER}\left(\bar{P}_{Q=6}\right)=10^{-9}
\end{aligned}
$$

Both bounds are selected to describe a received power range within measurable BER values. Higher BER values than for $\bar{P}_{R x}(Q=2)$ can hardly be measured with standard BER testing devices, as synchronization to the bit-pattern fails. The same applies for the upper bound: measuring lower BER than for $\bar{P}_{R x}(Q=6)$ can become impractical for low data-rate RFEs, due to very long integration times, during which the system characteristics may change e.g. due to thermal drift. Thus only the behavior between $\bar{P}_{Q=2}$ and $\bar{P}_{Q=6}$ is of practical interest and will be modelled for practical usefulness.

From (8) and (9) the ratio between Q-values becomes $q=3$ and the exponent $n$ is calculated as

$$
n=\frac{\ln 3}{\ln s} \text {, where } s=\frac{\bar{P}_{Q=6}}{\bar{P}_{Q=2}}
$$

Then (6) becomes

$$
Q\left(\bar{P}_{R x}\right)=2\left(\frac{\bar{P}_{R x}}{\bar{P}_{Q=2}}\right)^{n}
$$

where for clarity we omit any subindex letters for $x=2$ and $y=6$ from here on. Hence, the dependency of $Q$ versus the received power can be modelled as a function of three variables $Q=f\left(\bar{P}_{Q=2}, n, \bar{P}_{R x}\right)$. These parameters can be related to the analytical receiver models in Table I as shown under section IV.

To enable a rate-independent comparison of the quality of $Q\left(\bar{P}_{R x}\right)$, we normalize $\bar{P}_{Q=2}$ according to

$$
\bar{E}_{Q=2}=\frac{\bar{P}_{Q=2}}{r}[J]
$$

where $\bar{E}_{Q=2}$ is the required mean optical energy per data-bit in Joules [J] for $Q=2$. For wavelength-independent comparison, this sensitivity can be further expressed in mean photons-perbit $\bar{N}_{Q=2}$ by

$$
\bar{N}_{Q=2}=\bar{E}_{Q=2} / E_{p h}=\bar{E}_{Q=2} /(h \cdot c / \lambda)
$$

where $E_{p h}$ is the photon energy, $h$ is the Planck constant, $c$ is the speed of light and $\lambda$ is the wavelength. For an overview of various RFE sensitivities based on photons-per-bit see [16], fig. 61.

\section{ApPlicAtion to ANALYTICAl AND MEASURED RECEIVERS}

\section{A. Application to theoretical models}

Applying (11) to the analytical RFE-formulas in Table I, we find for the thermal noise limited RFE a linear dependency from received power, $n_{t}=1$, and therefore $s_{t}=3$, while the shot-noise limited homodyne-coherent BPSK is modeled by its square root relation to received power, $n_{s-B P S K}=0.5$, thus $s_{s-B P S K}=9$.

For the mixed shot- and thermal-noise dependent OOKAPD-receiver, from equation (4) we can find

$$
\bar{P}_{s t, \text { OOK }}=\frac{Q^{2} e F_{A} B}{R}+\frac{Q i_{n} \sqrt{B}}{R M}
$$


where $Q$ has been kept inside formula (14) for clarity, which finally has to be set to $Q=2$ to find the individual $\bar{P}_{Q=2}$. The $s$ parameter is then deduced for the power span $\left[\bar{P}_{Q=2}, \bar{P}_{Q=6}\right]$ as

$$
s_{s t}=3 \cdot\left(\frac{3 C_{s t, Q=2}+1}{C_{s t, Q=2}+1}\right)
$$

where the dimensionless constant $C_{\text {st }}$ is defined as

$$
C_{s t}=Q e M F_{A} \frac{\sqrt{B}}{i_{n}}
$$

For $C_{s t} \rightarrow 0$ the RFE is thermally limited, while for $C_{s t} \rightarrow \infty$ it is purely shot-noise limited.

Applying practical parameter values $\left(R=1 \mathrm{~A} / \mathrm{W}, M=20, F_{A}=8\right.$ [17], and $i_{n}=5.9 \mathrm{pA} / \sqrt{\mathrm{Hz}}$ as typical thermal noise density deduced from $\{11\}$ of Table III), we find $\bar{E}_{Q=2, t}=238 \mathrm{aJ}$, $\bar{E}_{Q=2, s-B P S K}=0.16 \mathrm{aJ}$, and $\bar{E}_{Q=2, s t}=14.4 \mathrm{aJ}$ for $r=1.25 \mathrm{Gbps}$, with $s_{s t}=4.1$. Figure 3 compares the ideal models of Table I with equation (11). All slopes are normalized to $\bar{P}_{Q=2}$.

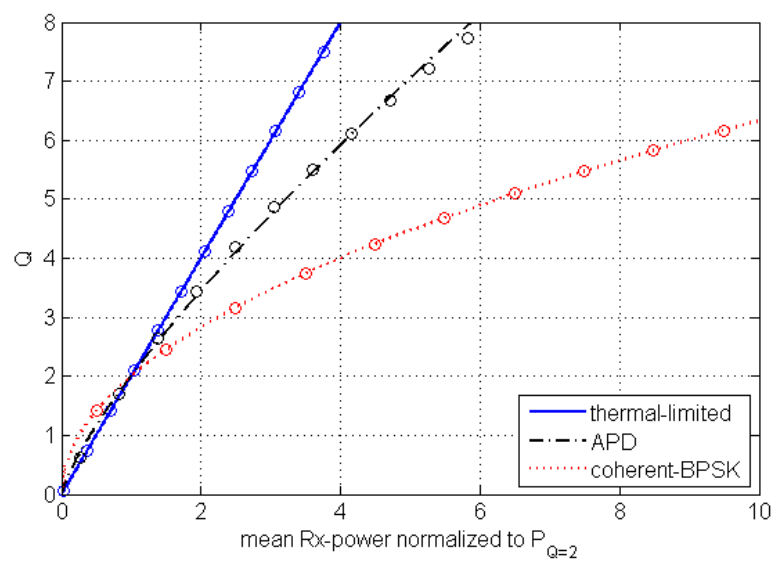

Fig. 3: Comparison of analytical formulas according to Table I (lines) with the modelling fit according equation (11) (circles).

\section{B. Application to measured performance values}

In this section, modelling fits according to equations (8)(11) are compared to the measured performance of three different RFE technologies.

Practical thermal limited RFEs [18], as well as labimplementations of coherent-homodyne BPSK-receivers [19] [20] agree well with their theoretical model formulas (2) and (3), while APD-receivers and high-bandwidth PIN RFEs exhibit a very individual behavior as can be seen in Figure 4 .

In Table III the model was applied to twelve measured RFE performances, where the root-mean-square relative fitting error (RMSRE) is provided as accuracy metric of the proposed model. The RMSRE of the fitting curve with respect to the measured data remains well below $10 \%$ for all analyzed RFEs.

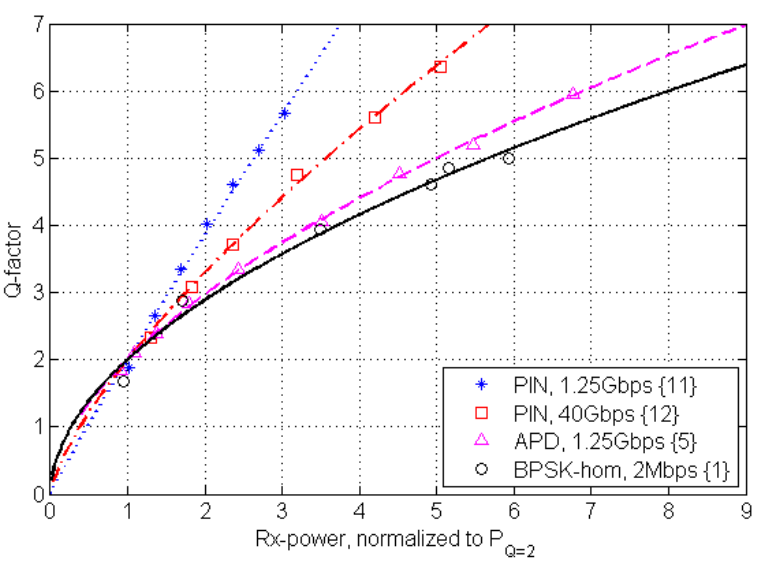

Fig. 4: Modelling of the Q-factor of measured RFE performances: thermal limited PIN-RFE $\{11\}$, high-bandwidth PIN-RFE $\{12\}$, APD-RFE $\{5\}$, and coherent homodyne BPSK $\{1\}$; sensitivities normalized to $P_{Q=2}$.

The model presented in this paper can describe any RFE behavior using equation (11). When enough measured data points are available, one can use a numerical fitting-tool directly, finding the exponent $n$ and the scaling factor $\bar{P}_{Q=2}$ which minimize the RMSRE. With only two measured points, as usually available from datasheets, one can completely define the RFE model (see $\{3\}$ of Table III). The reference values $\bar{P}_{Q=2}$ and $\bar{P}_{Q=6}$ can easily be related to any two measured values $\left(\bar{P}_{Q=x}, \bar{P}_{Q=y}\right)$ with equations (17) and using (7)

$$
\bar{P}_{Q=2}=\bar{P}_{Q=x}\left[\frac{2}{x}\right]^{1 / n_{x, y}} \quad ; \quad \bar{P}_{Q=6}=\bar{P}_{Q=y}\left[\frac{6}{y}\right]^{1 / n_{x, y}}
$$

From these reference power values $n$ is deduced using (10).

If no measured data is available one can choose values for $\bar{P}_{Q=2}$ and $s$ using typical parameter ranges provided in Table II and Figure 5. These values are derived from the fitted measured RFEs summarized in Table III. Typical APD receivers assume $s$ and $n$ values between the thermal-limited and shot-noise limited cases.

TABLE II

VALUE RANGES OF RECEIVER MODEL PARAMETERS

\begin{tabular}{ccc} 
Parameter & Typical Range & \multicolumn{1}{c}{ Explanation } \\
\hline $\bar{E}_{\mathrm{Q}=2}$ & 0.2 aJ. .700 aJ & sensitivity per bit in $10^{-18} \mathrm{~J}$ \\
$s$ & typical $3<\mathrm{s}<9$ & shot noise limited: $\mathrm{s}=9, \mathrm{n}=0.5$ \\
$n$ & typical $0.5<\mathrm{n}<1$ & thermal noise limited: $\mathrm{s}=3, \mathrm{n}=1$ \\
\hline \hline
\end{tabular}


Typical relations of absolute sensitivity $\bar{E}_{Q=2}$ and span are presented in Figure 5.

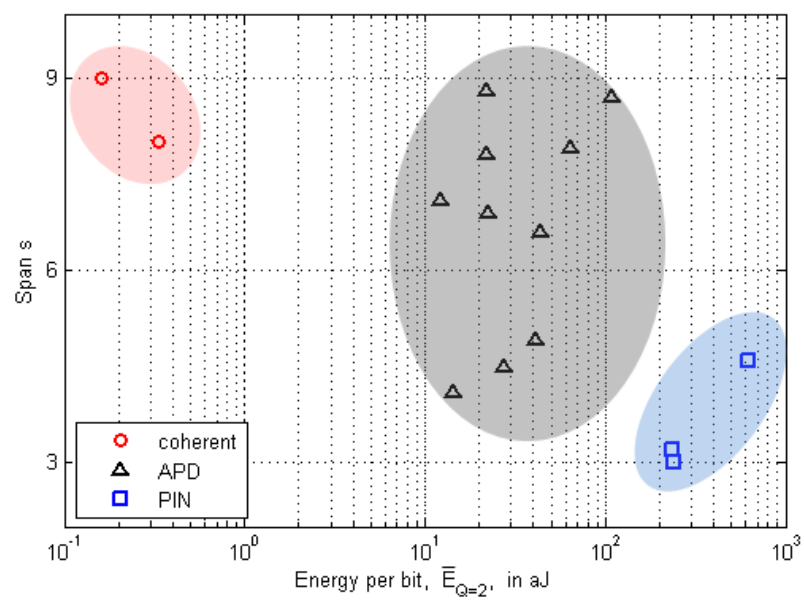

Fig. 5: Typical sensitivity-, and span-ranges for different types of binary optical RFEs, reflecting analytical values and measured data as given in Table III.

\section{SUMMARY}

We define a generally usable formalism to model optical receiver performance in terms of Q-factor, employing two main parameters $\bar{P}_{Q=2}$ and the exponent $n$ according to (10) and (11). $\bar{P}_{Q=2}$ directly indicates the lower usable sensitivity limit of the frontend and allows direct comparison of RFE sensitivity in terms of energy-per-bit in its normalized form $\bar{E}_{Q=2}$. The exponent $n$ defines the sensitivity behavior in the span $s$, or in other words defines the slope of the sensitivity curve. Span $s$ specifies the region in which the BERperformance changes from high BER to near error-free.

Theoretical as well as empirically observed sensitivity values can be modelled with our methodology without requiring receiver-type specific dedicated formalisms or employing specific physical parameters.

For any classical receiver $s$ lies between 3 and 9, where large values define a broader working-range. Values in between are observed with practical receivers when going from pure thermal noise (PIN-receivers) to including also signal-dependent noise sources (APDs, high-bandwidth PINs, heterodyning receivers). Table III presents the application of our methodology to various measured RFE-characteristics.

This methodology can e.g. beneficially be applied in simulating communication scenarios with high dynamic range - such as mobile Free-Space Optical Links with varying link distances or atmospheric scintillations - to optimize error correction mechanisms.

\section{ACKNOWLEDGMENT}

Measurement data of several InGaAs-APD RFEs were kindly provided by ViaLight Communications GmbH. The SILEX-Frontend $\{2\}$ was built by one of the authors under ESA-contract. 
TABLE III

Measured ReCeiver Model Parameters Summary, Sorted by mean Energy per Bit $\bar{E}_{Q=2}$

\begin{tabular}{|c|c|c|c|c|c|c|c|c|}
\hline No. & RFE-Type & data rate & $\bar{P}_{Q=2}$ & $s$ & $\bar{E}_{Q=2}$ & $\bar{N}_{Q=2}$ & $n$ & RMSRE \\
\hline$\{1\}$ & Homodyne BPSK SyncBit (1064nm) & 2Mbps & $0.672 \mathrm{pW}$ & 8.0 & $0.336 \mathrm{aJ}$ & 1.80 & 0.529 & $7.2 \%$ \\
\hline$\{2\}$ & APD-RFE-SILEX (820nm), M-fix & 4Mbps & $48.8 \mathrm{pW}$ & 7.1 & $12.2 \mathrm{aJ}$ & 50.3 & 0.558 & $4.3 \%$ \\
\hline$\{3\}$ & 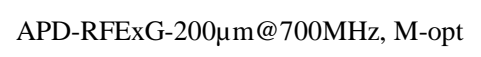 & 1.6Gbps & 35.3nW & 7.8 & $22.1 \mathrm{a} J$ & 172 & 0.536 & $0 \%$ \\
\hline$\{4\}$ & 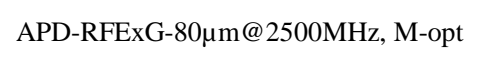 & 3.2Gbps & $71.2 \mathrm{nW}$ & 8.8 & $22.2 \mathrm{a} J$ & 173 & 0.507 & $2.1 \%$ \\
\hline$\{5\}$ & 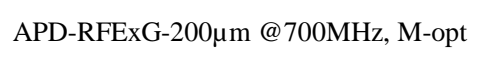 & $1.25 \mathrm{Gbps}$ & $28.1 \mathrm{nW}$ & 6.9 & $22.5 \mathrm{aJ}$ & 175 & 0.569 & $1.4 \%$ \\
\hline$\{6\}$ & commercial APD-TIA module, M-fix & 10Gbps & $273 n W$ & 4.5 & 27.3aJ & 213 & 0.729 & $6.3 \%$ \\
\hline$\{7\}$ & APD-RFE100@65MHz, M-opt & $125 \mathrm{Mbps}$ & $5.13 \mathrm{nW}$ & 4.9 & $41.0 \mathrm{aJ}$ & 320 & 0.692 & $2.8 \%$ \\
\hline$\{8\}$ & APD-RFE1G@650MHz filter, M-opt & $1.3 \mathrm{Gbps}$ & $57.1 \mathrm{nW}$ & 6.6 & 43.9aJ & 342 & 0.581 & $2.2 \%$ \\
\hline$\{9\}$ & APD-RFE1G@100MHz filter, M-opt & 200Mbps & $12.8 \mathrm{nW}$ & 7.9 & $64.0 \mathrm{aJ}$ & 499 & 0.529 & $3.0 \%$ \\
\hline$\{10\}$ & APD-RFE1G@20MHz filter, M-opt & 40Mbps & $4.36 \mathrm{nW}$ & 8.7 & 109aJ & 849 & 0.508 & $2.9 \%$ \\
\hline$\{11\}$ & commercial CWDM-PIN-receiver & $1.25 \mathrm{Gbps}$ & 296nW & 3.2 & 237aJ & $1.85 \mathrm{E} 3$ & 0.951 & $3.2 \%$ \\
\hline$\{12\}$ & ASK-PIN-RFE & 40Gbps & $24.9 \mu \mathrm{W}$ & 4.6 & $623 \mathrm{aJ}$ & $4.86 \mathrm{E} 3$ & 0.720 & $2.0 \%$ \\
\hline
\end{tabular}

All measured RFEs except $\{1\}$ and $\{2\}$ are tested with $1550 \mathrm{~nm}$ wavelength, $\mathrm{R}=1 \mathrm{~A} / \mathrm{W}$ is assumed, and PRBS is $2^{7}-1$ unless otherwise noted

M-fix: the multiplication factor $M$ of the APD is kept constant for all values of $\bar{P}_{R x}$

M-opt: $M$ is optimized for minimum BER at each measured $\bar{P}_{R x}$

RMSRE refers to the root-mean-square of the relative error between measured and fitted data-points of $Q$ vs $\bar{P}_{R x}$

$\{1\}$ homodyne-BPSK RFE with Syncbit-OPLL-System, at 1064nm laser wavelength, uncoded BER-performance [19][20]

\{2\} Si-APD receiver used at ESA's Optical Ground Station (Tenerife) [21], 500 $\mu \mathrm{m}$ diam. Si-APD, tested with 820nm signal wavelength

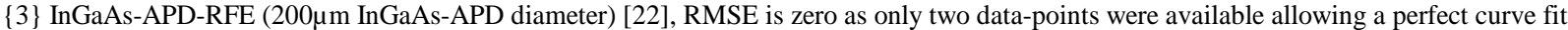

$\{4\}$ InGaAs-APD-RFE with 80 $\mu$ m detector diameter [22]

$\{5\}$ same as $\{3\}$ but measured with 1.25Gbps

\{6\} InGaAs-APD receiver bonded with TIA in one module, at 10Gbps, $M_{f i x}=5$, data taken from [23], PRBS $2^{23}-1$

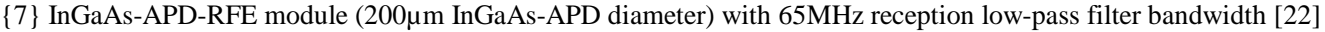

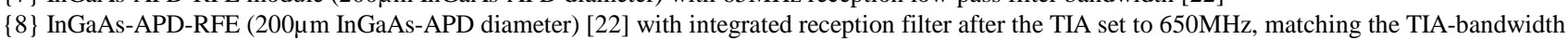

$\{9\}$ same APD-RFE as $\{8\}$ but with reception filter set to $100 \mathrm{MHz}$ (employing the LMH6518 as variable-bandwidth intermediate amplifier)

$\{10\}$ same APD-RFE as $\{8\}$ but with reception filter set to $20 \mathrm{MHz}$

\{11\} CWDM receiver module, with disabled squelch-function to enable measurements at high BER [18]

$\{12\}$ high-bandwidth direct detection InGaAs-PIN-RFE, BER-data taken from product specifications [24], PRBS not stated

\section{REFERENCES}

[1] M.A. Khalighi, M. Uysal, "Survey on Free Space Optical Communication: A Communication Theory Perspective", IEEE Communications Surveys \& Tutorials, Vol. 16 No. 4, 2014

[2] N. Perlot, T. De Cola, "Throughput Maximization of Optical LEO-Ground Links”, Free-Space Laser Comm. Technologies XXIV, San Francisco, USA, 2012

[3] F. Moll, "Experimental analysis of channel coherence time and fading behavior in the LEO-ground link." International Conference on Space Optical Systems and Applications, Kobe, Japan, May 2014

[4] M. Kubicek, H. Henniger, Z. Kolka, "Bit error distribution measurements in the atmospheric optical fading channel” In: Proceedings of SPIE -- Volume 6877.
Free-Space Laser Communication Technologies XX, San Jose, California, USA, 2008

[5] Andrews, L. and Phillips, R., Laser Beam Propagation through Random Media”, SPIE Press, 2nd edition, 2005

[6] D.J. Israel, B.L. Edwards, K.E. Wilson, J.D. Moore, “An Optical Communication Pathfinder for the Next Generation Tracking and Data Relay Satellite“, SpaceOps 2014 Conference, Pasadena, CA, 5-9 May 2014

[7] F. Heine, G. Mühlnikel, H. Zech, S. Philipp-May, R. Meyer, "The European Data Relay System, high speed laser based data links," Advanced Satellite Multimedia Systems Conference and the 13th Signal Processing for Space Communications Workshop (ASMS/SPSC), Livorno, Italy, 8-10 Sept. 2014

[8] H. Zech, F. Heine, M. Motzigemba, „Laser Communications Terminal: Product Status and Industrialization Process“, International Conference on 
Space Optical Systems and Applications, ICSOS-2014, Kobe, Japan, 7-9 May 2014

[9] G. P. Agrawal, "Fiber-Optic Communication Systems, Third Edition”, John Wiley \& Sons Inc., 2002

[10] Y. Yamamoto, "Fundamentals of Noise Processes", Cambridge University Press, 2004

[11]S.B. Alexander, "Optical Communication Receiver Design”, SPIE Tutorial Texts in optical engineering v. TT22, Bellingham 1997

[12] J. R. Barry, E. A. Lee, "Performance of Coherent Optical Receivers", Proceedings of the IEEE, 78, No. 8, 1990

[13] P.P. Webb, R.J. McIntyre, J. Conradi, "Properties of Avalanche Photodiodes", RCA Review Vol. 35, June 1974

[14] James N. Hollenhorst, „A Theory of Multiplication Noise”, IEEE Transactions on Electronic Devices, Vol. 37, No. 3, March 1990

[15] F. David, "Scintillation Loss in Free-Space Optic IM/DD Systems”, SPIE Free-Space Laser Communications Technologies XVI, Vol. 5338, 2004

[16]D.O. Caplan, "Laser communication transmitter and receiver design”, in: Free Space Laser Communications, p 109-246, Springer, New York, 2008

[17] “Opto-Semiconductor Handbook“, Hamamatsu Photonics, 2010

[18] APAC Opto El. Inc., "LS-42 CAU: CWDM 1550nm SMTransc.", data sheet, Hsinchu Hsien, 2009

[19] B. Wandernoth, "5 Photons/Bit Low Complexity 2Mbits/s BPSK Transmission Breadboard Experiment with Homodyne Receiver Applying Synchronization Bits and Convolutional Coding”, $20^{\text {th }}$ European Conference on Optical Communication, ECOC 1994

[20] B. Wandernoth, private communications, Feb. 2014

[21] A. Alonso, M. Reyes, Z. Sodnik, "Performance of satellite-to-ground communications link between ARTEMIS and the Optical Ground Station”, Proc. SPIE 5572, 2004

[22] ViaLight Communications GmbH, private com., 2014

[23] Kyosemi Corporation, "10Gbps APD-TIA Receiver KPDXA10G series”, data sheet, Kyoto, 2013

[24] SHF Comm. Tec. AG, "SHF 41210B, 40 Gbps Optical ASK Receiver”, data sheet, Berlin, 2008

Dirk Giggenbach received the Dipl.-Ing. (M.S.) degree in electrical engineering from the Technical University of Munich in 1994 and the Dr.-Ing. (Ph.D.) degree from the University of the German Federal Armed Forces at Munich in 2004.

He established and managed the Group "Optical Free-Space Communications" of the German Aerospace Center (DLR), at Site Oberpfaffenhofen near Munich. Between 2009 and 2014, he founded and built up the DLR spin-off company ViaLightCommunications $\mathrm{GmbH}$, developing aeronautical optical communication systems. In 2011 he was visiting researcher at the University of South Australia at Adelaide. Current research interests are the modelling of the optical feeder link to geostationary satellites and DWDM-technologies in free space optical communications at Tbps data rates.

Dr. Giggenbach is German delegate to CCSDS and regular reviewer for OSA and IEEE journals.

Ramon Mata Calvo received the double Dipl.-Ing. (M.S.) degree in telecommunications engineering from the Universitat Politècnica de Catalunya in Barcelona, Spain, and the Politecnico di Torino in Turin, Italy, in 2005. In 2009 he received the Dr.-Ing. (Ph.D.) degree from the the Politecnico di Torino in Turin, Italy.

In 2009, he joined the German Aerospace Center (DLR), at Site Oberpfaffenhofen near Munich. Since 2013 he has been managing the Group "Advanced Optical Technologies" of the Institute of Communications and Navigation in DLR. Current research interests are adaptive optics, and the modelling of the turbulent channel in free space optical communications. 